\title{
Diagnosis and treatment of ascites
}

Emmanuel A. Tsochatzis ${ }^{1}$, Alexander L. Gerbes ${ }^{2}$

1. UCL Institute for Liver and Digestive Health, Royal Free Hospital and UCL, London, UK

2. Medizinische Klinik und Poliklinik 2, Liver Centre Munich, Klinikum der LMU München-Grosshadern, University Hospital (KUM), Munich, Germany

Corresponding author: Alexander L. Gerbes, Medizinische Klinik und Poliklinik, Klinikum der LMU München-Grosshadern, University Hospital (KUM), Munich, Germany, email: Gerbes@med.uni-muenchen.de

Conflicts of interest: none

Financial support: none

Authors contributions: EAT and ALG both drafted the article, contibuted to the concept and design, critically revised for important intellectual content and approved before submission. 
Ascites is the most common complication of cirrhosis and its development is a sign of significant portal hypertension [1]. It becomes clinically detectable when in excess of 1.5 lt. Ascites in cirrhosis is the result of a vicious cycle involving arterial splanchnic vasodilation, decreased effective blood volume despite a compensatory increase in cardiac output, renal vasoconstriction with resulting sodium retention and finally extracellular fluid retention [2]. Systemic inflammation can exacerbate portal hypertension, contribute to the development of ascites and impair prognosis.

Detection of ascites should prompt medical history and clinical examination, laboratory investigations including full liver screen, a diagnostic tap (paracentesis) and abdominal imaging. Ascitic fluid should be sent for cell count, total protein, albumin, cytology and cultures in all patients and for LDH, cholesterol and amylase in selected cases. New onset of ascites in a patient with cirrhosis should prompt abdominal imaging to exclude portal vein thrombosis or hepatocellular carcinoma. A serum to ascites albumin gradient (SAAG) $\geq 1.1 \mathrm{~g} / \mathrm{dl}$ has $97 \%$ accuracy for diagnosis of ascites due to portal hypertension. Patients with a total protein of $<1.5 \mathrm{~g} / \mathrm{dl}$ and advanced liver disease should be considered for primary prophylaxis of spontaneous bacterial peritonitis (SBP) [3]. Usual clinical practice is to reserve primary prophylaxis for selected patients in the transplant waiting list. A neutrophil count (cut-off $250 / \mu$ ) should be obtained in each diagnostic tap to diagnose SBP. Patients with SBP should be treated empirically with a third generation cephalosporin in community-acquired infections or according to the local microbiological resistance profile in nosocomial infections (until culture results become available) together with albumin infusions (1.5 g/Kg on day 1 and $1 \mathrm{~g} / \mathrm{Kg}$ on day 3) [4, 5]. Once the 
episode is resolved, secondary prophylaxis should be instigated; the choice of antibiotic depends on the bacterial resistance in the ascites and is a quinolone in uncomplicated cases [6].

Once cirrhosis is confirmed as the cause of ascites, etiological treatment for the underlying liver disease should be considered and initiated as soon as possible as the patient might re-compensate with successful treatment (indicatively antivirals for hepatitis B, alcohol cessation in alcohol-related cirrhosis). Angiotensin-converting enzyme inhibitors and non-steroidal antiinflammatories should be stopped and aminoglycosides should be avoided as they can worsen ascites and/or induce renal failure. The initial treatment of mild/moderate ascites includes moderate sodium restriction to 80-120 $\mathrm{mmol} /$ day and the use of diuretics [6]. Spironolactone (100 mg/day) is the diuretic of choice, with or without $40 \mathrm{mg} /$ day of furosemide. Spironolactone monotherapy is recommended for first presentation of ascites, while combination with furosemide is preferred in recurrent ascites [6]. Diuretic treatment should be monitored and tailored according to weight loss (max 800g/day), kidney function and serum electrolytes. Random urinary electrolytes can be used to tailor and adjust diuretic treatment. Diuretics can be increased to a maximum dose of $400 \mathrm{mg} /$ day and $160 \mathrm{mg} /$ day for spironolactone and furosemide, respectively. Complications of diuretic treatment include acute kidney injury, electrolyte disorders, encephalopathy, muscle cramps and tender gynaecomastia; therefore patients should be monitored closely particularly when treatment is initiated or following dose increases. A $50 \%$ increase of creatinine values in 7 days or an absolute increase by $>26.5 \mu \mathrm{mol} / \mathrm{L}(0.3 \mathrm{mg} / \mathrm{dL})$ in 48 hours should prompt temporary discontinuation of diuretics or a dose 
reduction [6]. Diuretics should also be discontinued if severe hyponatraemia develops $(<120 \mathrm{mmol} / \mathrm{L})$.

Refractory ascites is defined as persistence of ascites despite sodium restriction and maximum dose or maximally tolerated dose of diuretics and is associated with a one-year mortality of over $40 \%$ [7]. Patients with refractory ascites, history of SBP or hepatorenal syndrome should be referred and worked up for liver transplantation if eligible. Large volume paracentesis (LVP) aiming to remove all fluid is the initial treatment of patients with large, recurrent or refractory ascites. No correction of coagulation is required for a paracentesis. In order to prevent the occurrence of circulatory dysfunction and renal impairment, $8 \mathrm{~g}$ of albumin should be administered intravenously after LVP for every liter of fluid removed if the total paracentesis volume is $>5$ liters [8].

The use of non-selective beta-blockers (NSBB) in patients with refractory ascites is controversially discussed. While NSBBs are associated with marked hypotension in patients with SBP, they seem to reduce mortality of patients with acute-on-chronic liver failure [9] or those with ascites waiting for liver transplantation [10]. Judicious use of NSBBs is therefore recommended in order to avoid hypotension and renal impairment.

TIPS is an alternative for recurrent or refractory ascites and can improve survival probability in eligible patients [11]. The presence of overt encephalopathy, serum bilirubin $>5 \mathrm{mg} / \mathrm{dL}$, portal vein thrombosis and cardiac insufficiency should be ruled out beforehand. In terms of laboratory parameters, a combination of a bilirubin level below $50 \mathrm{umol} / \mathrm{L} / 3 \mathrm{mg} / \mathrm{dL}$ and platelet count $>75,000 / \mathrm{mm}^{3}$ is associated with best outcomes [12]. If the patient is ineligible 
for TIPS or liver transplantation, then clinical trials, such as for the alpha pump system, are available in selected centers. 
[1] Tsochatzis EA, Bosch J, Burroughs AK. Liver cirrhosis. Lancet 2014;383:1749-1761.

[2] Arroyo V, Colmenero J. Ascites and hepatorenal syndrome in cirrhosis: pathophysiological basis of therapy and current management. Journal of hepatology 2003;38 Suppl 1:S69-89.

[3] Wiest R, Krag A, Gerbes A. Spontaneous bacterial peritonitis: recent guidelines and beyond. Gut 2012;61:297-310.

[4] Jalan R, Fernandez J, Wiest R, Schnabl B, Moreau R, Angeli P, et al. Bacterial infections in cirrhosis: a position statement based on the EASL Special Conference 2013. Journal of hepatology 2014;60:1310-1324.

[5] Piano S, Fasolato S, Salinas F, Romano A, Tonon M, Morando F, et al. The empirical antibiotic treatment of nosocomial spontaneous bacterial peritonitis: Results of a randomized, controlled clinical trial. Hepatology 2016;63:12991309.

[6] EASL clinical practice guidelines on the management of ascites, spontaneous bacterial peritonitis, and hepatorenal syndrome in cirrhosis. JHepatol 2010;53:397-417.

[7] Arroyo V, Gines P, Gerbes AL, Dudley FJ, Gentilini P, Laffi G, et al. Definition and diagnostic criteria of refractory ascites and hepatorenal syndrome in cirrhosis. International Ascites Club. Hepatology 1996;23:164-176.

[8] Bernardi M, Caraceni P, Navickis RJ, Wilkes MM. Albumin infusion in patients undergoing large-volume paracentesis: a meta-analysis of randomized trials. Hepatology 2012;55:1172-1181. 
[9] Mookerjee RP, Pavesi M, Thomsen KL, Mehta G, Macnaughtan J, Bendtsen F, et al. Treatment with non-selective beta blockers is associated with reduced severity of systemic inflammation and improved survival of patients with acuteon-chronic liver failure. Journal of hepatology 2016;64:574-582.

[10] Leithead JA, Rajoriya N, Tehami N, Hodson J, Gunson BK, Tripathi D, et al. Non-selective beta-blockers are associated with improved survival in patients with ascites listed for liver transplantation. Gut 2015;64:1111-1119.

[11] Salerno F, Camma C, Enea M, Rossle M, Wong F. Transjugular intrahepatic portosystemic shunt for refractory ascites: a meta-analysis of individual patient data. Gastroenterology 2007;133:825-834.

[12] Rossle M, Gerbes AL. TIPS for the treatment of refractory ascites, hepatorenal syndrome and hepatic hydrothorax: a critical update. Gut 2010;59:988-1000. 